Canad. Math. Bull. Vol. 22 (2), 1979

\title{
SEMILINEAR SECOND ORDER ELLIPTIC OSCILLATION
}

\author{
BY \\ C. A. SWANSON ${ }^{(1)}$
}

1. Introduction. These pages summarize recent progress on the oscillation problem for semilinear elliptic partial differential equations of the form

$$
\begin{gathered}
L(u ; x) \equiv \sum_{i, j=1}^{n} D_{i}\left[A_{i j}(x) D_{j} u\right]+B(x, u)=0 \\
x \in \Omega, \quad D_{i}=\partial / \partial x_{i}, \quad i=1, \ldots, n
\end{gathered}
$$

in unbounded domains $\Omega$ in $\boldsymbol{n}$-dimensional Euclidean space $\boldsymbol{R}^{\boldsymbol{n}}$. Our attention is restricted to the second order symmetric equation (1), and completeness is not attempted; the emphasis is on results obtained in the last five years.

Points in $R^{n}$ are denoted by $x=\left(x_{1}, \ldots, x_{n}\right)$ and the Euclidean length of $x$ is written $|x|$. The following notation will be used throughout:

$$
\begin{aligned}
S_{a} & =\left\{x \in R^{n}:|x|=a\right\}, \quad a>0 ; \\
G_{a} & =\left\{x \in R^{n}:|x|>a\right\} ; \\
\Omega_{a} & =\Omega \cap G_{a} ; \\
G(a, b) & =\left\{x \in R^{n}: a<|x|<b\right\} .
\end{aligned}
$$

The measure on $S_{\mathrm{r}}$ and $S_{1}$ will be denoted by $s$ and $\omega$, respectively: $d s=$ $r^{n-1} d \omega$. Our assumptions below on the functions $B$ and $A_{i j}, i, j=1, \ldots, n$ in

(1) could be relaxed somewhat, but are typical of those in [28-31, 38-41].

(A) Each $A_{i j}$ in (1) is a real-valued function of class $C^{1}(\Omega)$, and the matrix $A=\left(A_{i j}\right)$ is symmetric and positive definite in $\Omega$ (ellipticity condition);

(B $\left.B_{1}\right) B(x, t)$ is continuous in $\Omega \times R^{1}$ with $B(x,-t)=-B(x, t)$ for all $x \in \Omega, t>0$;

$\left(\mathrm{B}_{2}\right) B(x, t) \geq p(x) \phi(t)$ for all $x \in \Omega$ and for all $t \geq 0$, where $p$ is continuous in $\Omega$ and $\phi$ is a continuously differentiable function in $[0, \infty)$ with $\phi(t)>0$ in $(0, \infty)$.

Assumptions $\left(\mathrm{B}_{1}\right)$ and $\left(\mathrm{B}_{2}\right)$ need to be strengthened or modified for many of the theorems stated in $\$ \S 3-7$.

A function $f: \Omega \rightarrow R^{1}$ is called oscillatory in $\Omega$ whenever $f(x)$ has a zero in

This paper is one of a series of survey papers written at the invitation of the Editors of the Canadian Mathematical Bulletin.

(1) The University of British Columbia, Vancouver, B.C. V6T $1 \mathrm{~W} 5$. 
$\Omega_{a}$ for all $a>0$. An elliptic equation (1) (or inequality $u L(u ; x) \leq 0$ ) is called oscillatory in $\Omega$ whenever every solution of the equation (or inequality, respectively) is oscillatory in $\Omega$. The concept of nodal oscillation (or strong oscillation) has frequently been considered $[2,14-16,22,33,34,38,40]$ in the special case that (1) has the linear form (2) below, i.e. $B(x, t)$ has the form $p(x) t$. A nonempty bounded domain $M \subset \Omega$ is called a nodal domain of a nontrivial solution $u$ of (2) if and only if $u(x)=0$ for all $x \in \partial M$. Equation (2) is defined to be nodally oscillatory in $\Omega$ if and only if there exists a nontrivial solution $u_{a}$ of (2) with a nodal domain contained in $\Omega_{a}$ for all $a>0$. Otherwise stated, (2) is nodally oscillatory in $\Omega$ if and only if the Dirichlet problem $L(u ; x)=0$ in $M, u=0$ on $\partial M$ is not uniquely solvable in some nonempty bounded domain $M$ in $\Omega_{a}$ for all $a>0$. If $M \subset \Omega_{a}$ is a nodal domain of a nontrivial solution $u_{a}$ of (2), then every solution $u$ of (2) has a zero at some point in $\bar{M}$ by the $n$-dimensional version of Sturm's separation theorem [37]. Incidentally, the stronger conclusion that every solution of (2) except a constant multiple of $u_{a}$ has a zero at some point in $M$, without any boundary regularity hypotheses whatsoever, was proved in 1978 by Heywood, Noussair, and the writer [17]. It follows that a linear equation (2) is oscillatory in an unbounded domain $\Omega$ if it is nodally oscillatory in $\Omega$.

The converse of the last statement also is true provided the coefficients in (2) are sufficiently regular; Allegretto [2] proved it in 1974 under the following hypotheses: (i) $p \in C^{3 m}(\bar{M})$; (ii) Each $A_{i j} \in C^{3 m+1}(\bar{M})$; and (iii) $L$ is uniformly elliptic in $M$ for every bounded subdomain $M \subset \Omega$, where

$$
m=\left[\frac{1}{2}\left[\frac{n+6}{2}\right]\right]
$$

and $[\beta]$ denotes the largest integer not exceeding $\beta$. Allegretto showed how to construct a positive solution of (2) in $\Omega_{a+\varepsilon}$ for any $\varepsilon>0$ if the Dirichlet problem for (2) is uniquely solvable in every bounded domain $M$ in $\Omega_{a}$ for some $a>0$. This construction implies Allegretto's equivalence theorem: Nodal oscillation and (weak) oscillation of sufficiently regular symmetric linear elliptic equations are equivalent. We note that the regularity requirements are quite severe, but can be weakened according to Allegretto [2] and Piepenbrink [34]; the latter states that hypotheses (i) and (ii) can be replaced by $p \in C^{2 m}$ and each $A_{i j} \in C^{2 m+1}$, respectively, e.g. $p \in C^{4}$ if $n=2,3,4$, or 5. A related theorem was stated earlier by Kuks [22, Theorem 3] without proof and with incomplete hypotheses. Allegretto [2], Glazman [14], and Piepenbrink [33, 34] have all emphasized the relationship between nonoscillation of $L$ and the nature of the lower part of the spectrum of the selfadjoint realization of $-L$. Allegretto [2] gives the interesting example

$$
L(u ; x)=\sum_{i=1}^{2}\left[D_{i}\left(x_{i}^{-2} D_{i} u\right)+2 x_{i}^{-4} u\right]
$$


in the unit square $\Omega=\left\{x \in R^{2}: 0<x_{i}<1 ; i=1,2\right\}$ to illustrate that some kind of global regularity assumptions are needed, even for a regular bounded domain, in order that the existence of a positive solution of (2) in $\Omega$ implies the unique solvability of the Dirichlet problem in every subdomain of $\Omega$. In fact, $u(x)=x_{1} x_{2}$ is a positive solution in $\Omega$ while $u_{n}(x)=\left(x_{1}^{2}-x_{1} / n\right)\left(x_{2}^{2}-x_{2} / n\right)$ is a nontrivial solution of $L\left(u_{n} ; x\right)=0$ in $M, u_{n}=0$ on $\partial M$ for the subdomain

$$
M=\left\{x \in \Omega: 0<x_{i}<\frac{1}{n} ; i=1,2\right\}, \quad n=2,3, \ldots,
$$

and consequently the Dirichlet problem does not have a unique solution in all subdomains of $\Omega$.

$\$ 2$ contains a brief description of oscillation criteria for linear elliptic equations, dating back to a result of Glazman [14], thereby extending to $\boldsymbol{R}^{\boldsymbol{n}}$ various one-dimensional criteria of Kneser [37], Leighton [24], Moore [25], and others. The existence of positive solutions of (1) in exterior domains is the main topic of $\S 3$. In the case of the Emden-Fowler equation, for which $B(x, t)$ in (1) has the form $p(x) t^{\gamma}, \gamma>0$, specific criteria for the existence of a positive solution are used in $\S \S 5$ and 6 to generate necessary and sufficient conditions for (1) to be oscillatory in both the superlinear case $(\gamma>1)$ and the sublinear case $(0<\gamma<1)$. These results extend theorems of Atkinson [7] and Belohorec $[8,9,44]$ for ordinary differential equations to $n$ dimensions, and constitute the only known characterizations of oscillatory partial differential equations at this date. $\$ 3$ contains specific upper and lower bounds for positive solutions, and also a criterion for the existence of a bounded positive solution as a consequence of Nehari's one-dimensional criterion [26]. Some recent superlinear oscillation criteria [29] arise from a Riccati-type transformation (Lemma 11) together with a priori lower bounds for positive solutions of (1) (Lemma 9), as described in $\$ 4$. The method of spherical means [28] is used in $\$ 5$ to generate sharp oscillation criteria for Schrödinger equations; in fact, these are characterizations of oscillatory equations in some cases [30, 31]. A brief discussion of perturbed linear equations, without sign restrictions on the coefficient functions, is given in $\$ 7$, and some typical oscillation theorems in arbitrary unbounded domains are described in $§ 8$.

2. Linear equations. The first oscillation theorems for elliptic equations were obtained by Glazman [14], Headley [15, 16], Kreith [19, 20], Kreith and Travis $[21]$, and the author $[16,38-41]$ in the linear case

$$
L(u ; x)=\sum_{i, j=1}^{n} D_{i}\left[A_{i j}(x) D_{j} u\right]+p(x) u=0,
$$

where $p$ and $\left(A_{i j}\right)$ are as in assumptions $(\mathrm{A})$ and $\left(\mathrm{B}_{2}\right)$. Some of these results will now be described in the case that the unbounded domain $\Omega$ is an exterior domain, i.e. there exists a positive number $a$ such that $G_{a} \subset \Omega$. 
Let $\lambda(x)$ denote the (necessarily positive) largest eigenvalue of the matrix $\left(A_{i j}(x)\right)$ and let $f$ be any piecewise $C^{1}$ function in $(0, \infty)$ satisfying $f(r) \geq$ $\max _{|x|=r} \lambda(x), 0<r<\infty$. Also let $g(r)=\min _{|x|=r} p(x), 0<r<\infty$. Then [16] equation (2) is nodally oscillatory in an exterior domain $\Omega \subset R^{n}$ if either

$$
\int_{a}^{\infty} \frac{d r}{r^{n-1} f(r)}=+\infty \text { and } \int_{a}^{\infty} r^{n-1} g(r) d r=+\infty
$$

or

$$
\int_{a}^{\infty} \frac{d r}{r^{n-1} f(r)}<\infty \text { and } \int_{a}^{\infty} r^{n-1} h_{n}^{\mu}(r) g(r) d r=+\infty
$$

for some $a>0$ and $\mu>1$, where

$$
h_{n}(r)=\int_{r}^{\infty} \frac{d i}{t^{n-1} f(t)}
$$

If $n=1$, the conditions ( 3 ) reduce to the Leighton-Wintner oscillation criterion for an ordinary linear equation [24, 43] while (4) reduce to Moore's criteria [25]. If $\lambda(x)$ is bounded in $\Omega$, additional nodal oscillation criteria [16] are

$$
\begin{gathered}
\int_{a}^{\infty} r g(r) d r=+\infty \quad \text { if } \quad n=2, \\
\int_{a}^{\infty} r^{1-\delta} g(r) d r=+\infty \quad \text { if } \quad n \geq 3,
\end{gathered}
$$

for some positive numbers $a$ and $\delta$; and

$$
\liminf _{r \rightarrow \infty} r^{2} g(r)>\lambda_{0}(n-2)^{2} / 4
$$

where $\lambda_{0}$ is an upper bound on $\lambda(x)$ in $\Omega$. Condition (7) reduces to Glazman's criterion [14] if $\left(A_{i j}\right)$ in (2) is the identity matrix, and becomes Kneser's classical criterion in one dimension. The results (3)-(7) are proved in [16], and in fact are extended to unbounded domains which are not necessarily exterior domains: it is merely required that $\Omega$ be large enough at $\infty$ to contain a cone $C_{\alpha}=\left\{x \in R^{n}: x^{i} \geq|x| \cos \alpha\right\}$ for some $i, 1 \leq i \leq n$, and some $\alpha$ in $(0, \pi]$. Recently Noussair and the author [29] have sharpened and extended (5) and (6) as indicated below. For these results, $p_{M}(r)$ denotes the spherical mean of $p$ over the sphere $S_{r}$ :

$$
p_{M}(r)=\frac{1}{\omega\left(S_{1}\right)} \int_{S_{1}} p(x) d \omega
$$


where $\omega$ denotes the measure on $S_{1}$. The boundedness of the largest eigenvalue $\lambda(x)$ of $\left(A_{i j}(x)\right)$ is weakened to

$$
\begin{gathered}
\max _{|x|=r} \lambda(x) \leq C[\log (\log r)]^{\delta}, \quad r>e, \quad \text { if } \quad n=2 ; \\
\max _{|x|=r} \lambda(x) \leq C(\log r)^{\delta}, \quad r>1, \quad \text { if } \quad n \geq 3,
\end{gathered}
$$

for some numbers $C>0$ and $\delta, 0<\delta \leq 1$.

THEOREM 1. The linear inequality $u L(u ; x) \leq 0$ is oscillatory in an exterior domain $\Omega$ in $R^{2}, R^{n}(n \geq 3)$ if (9), (10) hold, respectively, and there exists a positive number a such that

$$
\begin{aligned}
& \int_{a}^{\infty}\left[r \log r p_{M}(r)-\frac{f(r)}{4 r \log r}\right] d r=+\infty \quad \text { if } \quad n=2 ; \\
& \int_{a}^{\infty}\left[r p_{M}(r)-\frac{(n-2)^{2} f(r)}{r}\right] d r=+\infty \quad \text { if } \quad n \geq 3 .
\end{aligned}
$$

These criteria are also nodal oscillation criteria if $p$ and the $A_{i j}$ in (2) are sufficiently regular because of Allegretto's equivalence theorem [2], as described in $\$ 1$. In the case of a constant matrix $\left(A_{i j}\right)$ in (2), conditions (9) and (10) hold automatically, and hence (11) and (12) are oscillation criteria if $f(r)$ is replaced by the (constant) largest eigenvalue $\lambda$ of $\left(A_{i j}\right)$. Another oscillation criterion obtained in [29] is

$$
\int_{\Omega} p(x) d x=+\infty \text { and } \int_{a}^{\infty} \frac{r^{1-n} d r}{f(r)}=\infty
$$

for some $a>0$. Positivity of $p(x)$ throughout $\Omega$ is not required for any of these results.

The sharpness of (11) and (12) can be seen by considering the special case that (2) is a radial Schrödinger equation $\Delta u+p_{M}(r) u=0$; then (11) and (12) are known [37] to be sharp one-dimensional criteria for the damped ordinary equation arising from separation of variables. Of course (11) and (12) do not characterize oscillatory equations (2): No characterizations are known even for ordinary linear equations. It is interesting to compare (11) with the characterization (32) in Theorem 19 (\$5) of oscillatory superlinear equations (2) of the special Emden-Fowler type (14).

3. Existence of positive solutions. Although the existence theorems below are true for the general semilinear elliptic equation (1), by essentially the same proofs, we shall state them for the Schrödinger equation

$$
L(u ; x)=\Delta u+B(x, u)=0, \quad x \in \Omega
$$

and in particular the Emden-Fowler equation

$$
\Delta u+p(x) u^{\gamma}=0, \quad x \in \Omega, \quad \gamma>0
$$


since the interesting applications are in these special cases. In particular, necessary and sufficient conditions for (14) to be oscillatory are generated in both the superlinear case $(\gamma>1)$ and the sublinear case $(0<\gamma<1)$. These are the only known characterizations of oscillatory partial differential equations, extending well-known theorems of Atkinson [7] and Belohorec $[8,44]$ to $n$ dimensions.

For a bounded domain $M \subset R^{n}$, the Hölder norms of functions $u: \bar{M} \rightarrow R^{1}$ are defined (as usual) by [23]

$$
\begin{aligned}
&\|u\|_{\alpha, \bar{M}}=\sup _{\substack{x, y \in M \\
x \neq y}} \frac{|u(x)-u(y)|}{|x-y|^{\alpha}} \\
&\|u\|_{m+\alpha, \bar{M}}=\sum_{|i|=m}\left\|D^{i} u\right\|_{\alpha, \bar{M}}+\sum_{|i| \leq m} \quad \sup _{x \in \bar{M}}\left|D^{i} u(x)\right|, \\
& 0<\alpha<1, \quad m=1,2, \ldots,
\end{aligned}
$$

where $i$ denotes a multi-index of length $|i|$. The Hölder space $C^{m+\alpha}(\bar{M})$ is the set of all continuous real-valued functions on $\bar{M}$ such that $\|u\|_{m+\alpha, \bar{M}}$ is finite, $0<\alpha<1, m=0,1,2, \ldots$

Equation (13) is to be considered in an exterior domain $\Omega$ in $R^{n}$ under the hypotheses below.

(R) Regularity hypothesis. $B \in C^{\alpha}(\bar{M} \times J)$, for some $\alpha$ in $0<\alpha<1$, for every bounded domain $M \subset \Omega$ and for every interval $J=[0, b], b>0$.

(MN) Monotony AND NONNEGativity hypotheses. $0 \leq B\left(x, t_{1}\right) \leq B\left(x, t_{2}\right)$ for all $t_{1}, t_{2}$ satisfying $0 \leq t_{1} \leq t_{2}$ and for all $x \in \Omega$.

Defintrion. A solution of $L(u ; x)=0$ in an exterior domain $\Omega$ is a function $u \in C^{2+\alpha}(\bar{M})$ for every bounded subdomain $M \subset \Omega$, with $\alpha$ as in hypothesis $(R)$, such that $L(u ; x)=0$ for all $x \in \Omega$. A solution of $L(u ; x) \leq 0$ or $L(u ; x) \geq 0$ is defined similarly.

THEOREM 2 [30, 31]. Let $L, \Omega$ and $\alpha$ be as above and suppose $a$ is a positive number such that $G_{a} \subset \Omega$. If there exists a positive (uniformly positive, respectively) solution $v(x)$ of $L(v ; x) \leq 0$ in $G_{a}$, then equation (13) has a positive (uniformly positive) solution $u(x)$ in $G_{a}$. If in addition there exists a positive solution of $L(w ; x) \geq 0$ in $G_{a}$ such that $w(x) \leq v(x)$ throughout $G_{a} \cup S_{a}$, then (13) has a solution $u(x)$ satisfying $w(x) \leq u(x) \leq v(x)$ throughout $G_{a} \cup S_{a}$.

An essential part of the proof is the inductive construction of a nonincreasing sequence of functions $u_{j}$ in $G_{a} \cup S_{a}$ which are solutions of (13) in annular subdomains $\left\{x \in R^{n}: a<|x|<a+j\right\}, j=0,1,2, \ldots$ This can be done by means of the maximum principle [35] and a theorem of Amann [6, p. 142], providing conditions for the existence of a solution $u_{j}$ of (12) in a bounded domain which is squeezed between a subsolution and a supersolution. Then the sequence is 
shown [30] to converge to a solution of (13) in $G_{a}$ by means of Sobolev embedding [23], classical Schauder estimates [12, p. 335], and $L^{p}$ estimates developed by Agmon, Douglis, and Nirenberg [1, p. 704].

Corollary 3. A necessary and sufficient condition for the existence of a positive solution of (13) in an exterior domain $G_{a}$ is the existence of a positive supersolution of (13) in $G_{a}$.

COROllary 4. For arbitrary $\varepsilon$ in $(0, n-2), n \geq 3$, (13) has a solution $u(x)$ in $G_{a} \subset R^{n}$, for some $a>0$, satisfying the inequalities

$$
|x|^{2-n} \leq u(x) \leq|x|^{2-n+\varepsilon}, \quad x \in G_{a}
$$

if

$$
\limsup _{r \rightarrow \infty} r^{n-\varepsilon} \max _{|x|=r} B\left(x,|x|^{2-n+\varepsilon}\right) \leq \varepsilon(n-2-\varepsilon) .
$$

For the Emden-Fowler equation (14), condition (15) reduces to

$$
\limsup _{r \rightarrow \infty} r^{b} P(r) \leq \varepsilon(n-2-\varepsilon), \quad n \geq 3,
$$

where

$$
P(r)=\max _{|x|=r} p(x), \quad b=n-(n-2) \gamma+(\gamma-1) \varepsilon .
$$

COROllaRy 5. For arbitrary $\varepsilon>0$ and $K_{1}, K_{2}$ with $0<K_{1}<K_{2}$, if

$$
\limsup _{r \rightarrow \infty} r^{2+\varepsilon} \max _{|x|=r} B\left(x, K_{2}\right) \leq \varepsilon^{2},
$$

then there exists $a>0$ such that (13) has a solution $u(x)$ in $G_{a} \subset R^{2}$ satisfying

$$
K_{1} \leq u(x) \leq K_{2}-r^{-\varepsilon} .
$$

In particular (13) has a positive bounded solution in $G_{a}$ if (15) or (16) is satisfied. A stronger result is obtained for (14) on the basis of a one-dimensional criterion of Nehari [26, p. 103]; this is stated as the next Corollary.

Corollary 6. For $\gamma>0$, the Emden-Fowler equation (14) has a bounded positive solution in an exterior domain in $R^{n}$ if $p$ is a nonnegative function in $C^{\alpha}(\bar{M}), 0<\alpha<1$, for every bounded subdomain $M \subset R^{n}$, and if

$$
\begin{gathered}
\int_{c}^{\infty} r \log r P(r) d r<\infty, \quad n=2 \\
\int_{c}^{\infty} r^{\sigma} P(r) d r<\infty, \quad n \geq 3,
\end{gathered}
$$

for some $c>0$, where $\sigma=(n-1)-\gamma(n-2)$. 
It is shown in [30] that nonoscillation criteria for (13) or (14) follow easily from Theorem 2. We illustrate the procedure below by proving that (17), (18) are nonoscillation criteria for (14) in the superlinear case $\gamma>1$.

LEMMA 7. In (14) suppose that $\gamma>0$, that $p(x)$ is nonnegative in an exterior domain $\Omega \subset R^{n}$ with $p \in C^{\alpha}(\bar{M})$ for every bounded domain $M \subset \Omega$, and that $P \in C^{\alpha}[a, b]$ for all $b>a, 0<\alpha<1$, where $P(r)=\max p(x)$ on $|x|=r$, as before. Then a sufficient condition for (14) to be nonoscillatory in $\Omega$ is the existence of a positive solution $\rho \in C^{2+\alpha}[a, b]$ of the ordinary differential equation (19) below for some number $a>0$ and for all $b>a$ :

$$
\frac{d}{d r}\left(r^{n-1} \frac{d \rho}{d r}\right)+r^{n-1} P(r) \rho^{\gamma}(r)=0, \quad r>0 .
$$

Proof. The function $v$ in $G_{a}$ defined by $v(x)=\rho(r), r=|x| \geq a$ satisfies (in the case that (13) reduces to (14))

$$
\begin{aligned}
r^{n-1} L(v ; x) & =\frac{d}{d r}\left(r^{n-1} \frac{d \rho}{d r}\right)+r^{n-1} p(x) \rho^{\gamma}(r) \\
& \leq \frac{d}{d r}\left(r^{n-1} \frac{d \rho}{d r}\right)+r^{n-1} P(r) \rho^{\gamma}(r),
\end{aligned}
$$

and hence $L(v ; x) \leq 0$ for all $x \in G_{a}$ by (19). Then Theorem 2 shows that (14) has a positive solution $u(x)$ in $G_{a}$.

THEOREM 8. Under the hypotheses on $p$ and $P$ in Lemma 7, (17) and (18) are sufficient conditions for the superlinear (i.e. $\gamma>1$ ) Emden-Fowler equation (14) to be nonoscillatory in an exterior domain $\Omega$ in $R^{2}$ and $R^{n}, n \geq 3$, respectively.

Proof. For $n=2$, Liouville's change of variables $r=e^{s}, h(s)=\rho\left(e^{s}\right)$ transforms (19) into

$$
h^{\prime \prime}(s)+e^{2 s} P\left(e^{s}\right) h^{\gamma}(s)=0, \quad \gamma>1,
$$

which has a positive solution $h(s)$ for sufficiently large $s$ if there is a positive number $s_{0}$ such that

$$
\int_{s_{0}}^{\infty} s e^{2 s} P\left(e^{s}\right) d s<\infty
$$

by Atkinson's theorem [7]. Since (20) is equivalent to (17), it follows that (17) is sufficient for (19) (when $\gamma>1, n=2$ ) to have a positive solution $\rho(r)$ in $[a, \infty)$ for some $a>0$. Since $P \in C^{\alpha}[a, b]$ for all $b>a, 0<\alpha<1$, standard regularity theorems [23] show that $\rho \in C^{2+\alpha}[a, b]$ for all $b>a$. The conclusion in the case $n=2$ then follows from Lemma 7. The case $n \geq 3$ is quite similar and is described in [30]. 
4. Superlinear equations. We now return our attention to the general semilinear elliptic equation (1) under the assumptions stated in $\S 1$. The following additional notation will be required:

$$
\begin{aligned}
L_{0}(u ; x) & =\sum_{i, j=1}^{n} D_{i}\left[A_{i j}(x) D_{j} u\right] \\
\rho(r) & =\sup _{|x|=r} \mid L_{0}(|x| ; x)\left[\sum_{i, j} A_{i j}(x) D_{i}|x| D_{j}|x|\right]^{-1} ; \\
S(r ; b) & =\int_{r}^{b} \exp \left[\int_{a}^{t}-\rho(s) d s\right] d t, \quad a \leq r \leq b \\
R(r ; b) & =S(r ; b) / S(a ; b) \\
R(r) & =\lim _{b \rightarrow \infty} R(r ; b) \\
U(a) & =\inf _{|x|=a} u(x) .
\end{aligned}
$$

LEMMA 9. Every positive solution $u$ of $L_{0}(u ; x) \leq 0$ for $|x| \geq a$ satisfies the inequality $u(x) \geq U(a) R(|x|)$ for $|x| \geq a$.

If $B(x, u)$ in (1) is nonnegative for all $x \in \Omega$ and for all $u>0$, Lemma 9 gives an a priori lower bound $U(a) R(|x|)$ on any positive solution $u(x)$ of $L(u ; x) \leq 0$ in $\Omega$, and enables us to deduce superlinear oscillation criteria from linear criteria. Lemma 9 is proved in [29] as a consequence of the Hopf maximum principle [35]. Related results have been derived and exploited by Allegretto [3]. In the superlinear case, assumptions $(A),\left(B_{1}\right)$, and $\left(B_{2}\right)$ will be augmented by the following additional ones:

SUPERLINEAR ASSUMPTIONS

$\left(\mathrm{SL}_{1}\right) p$ in assumption $\left(\mathrm{B}_{2}\right)$ is everywhere nonnegative in $\Omega$;

$\left(\mathrm{SL}_{2}\right) \psi(t)=\phi(t) / t$ is nondecreasing for all $t>0$.

COMPARISON THEOREM 10. Under assumptions $(\mathrm{A}),\left(\mathrm{B}_{1}\right),\left(\mathrm{B}_{2}\right),\left(\mathrm{SL}_{1}\right)$, and $\left(\mathrm{SL}_{2}\right)$, the superlinear inequality $u L(u ; x) \leq 0$ is oscillatory in an exterior domain $\Omega$ in $\boldsymbol{R}^{\boldsymbol{n}}$ if the linear inequality

$$
L_{0}(u ; x)+p(x) \psi(\varepsilon R(|x|)) u \leq 0
$$

has no eventually positive solution in $\Omega$ for any positive number $\varepsilon$.

Proof. If $u(x)$ were a positive solution of $u L(u ; x) \leq 0$ throughout $G_{a}$ for some $a>0$ (chosen large enough so that $G_{a} \subset \Omega$, without loss of generality), then

$$
\begin{aligned}
0 & \geq L_{0}(u(x) ; x)+B(x, u(x)) \\
& \geq L_{0}(u(x) ; x)+p(x) \psi(u(x)) u(x) \\
& \geq L_{0}(u(x) ; x)+p(x) \psi(U(a) R(|x|)) u(x)
\end{aligned}
$$


by Lemma $9,\left(\mathrm{~B}_{2}\right),\left(\mathrm{SL}_{1}\right)$, and the nondecreasing hypothesis $\left(\mathrm{SL}_{2}\right)$ on $\psi$. Thus $u(x)$ satisfies (21) with $\varepsilon=U(a)>0$, and cannot be eventually positive by hypothesis. Similarly a solution of $L(u ; x) \geq 0$ cannot be everywhere negative in $G_{a}$.

In [29] Noussair and the writer utilized a generalized Riccati-type transformation [36] defined in terms of an arbitrary positive absolutely continuous function $\alpha$ in $[0, \infty)$. This transformation maps positive $C^{1}$ scalar functions $u$ in $\Omega$ into $n$-vector functions $w$ defined by

$$
w(x)=-\alpha(|x|)[\phi(u(x))]^{-1}(A \nabla u)(x)
$$

Matrix notation is used in (22) and below: In particular $A^{-1}$ denotes the inverse of the matrix $A=\left(A_{i j}\right)$ and $*$ denotes the transpose.

LEMMA 11. If $u$ is a positive-valued solution of $L(u ; x) \leq 0$ in $\Omega$, under assumptions $(\mathrm{A}),\left(\mathrm{B}_{1}\right)$, and $\left(\mathrm{B}_{2}\right)$, then the $n$-vector function $w$ given by (22) satisfies the Riccati inequality

$$
\operatorname{div} w(x) \geq \alpha(r) p(x)+\frac{\phi^{\prime}(y(x))}{\alpha(r)}\left(w^{*} A^{-1} w\right)(x)+\frac{\alpha^{\prime}(r)}{\alpha(r)}\left(w^{*} v\right)(x),
$$

where $\nu(x)=x / r$, is the outward unit normal to $S_{r}, r=|x|$.

The proof is given in [29]. In the case of the linear inequality (21), the Riccati inequality (23) reduces to

$$
\operatorname{div} w(x) \geq \alpha(r) p(x) \psi(\varepsilon R(|x|))+\frac{\left(w^{*} A^{-1} w\right)(x)}{\alpha(r)}+\frac{\alpha^{\prime}(r)}{\alpha(r)}\left(w^{*} \nu\right)(x) .
$$

By the choice $\alpha(r)=\log r(r>1)$ for $n=2$ and $\alpha(r)=r^{2-n}(r>0)$ for $n \geq 3$ in (24), the following results are obtained in [29] by a modification of Coles' one-dimensional procedure [10].

THEOREM 12. Under the assumptions of Theorem 10, the superlinear inequaltiy $u L(u ; x) \leq 0$ is oscillatory in an exterior domain $\Omega$ of $R^{2}$ if (9) holds and there exists a positive number a such that

$$
\int_{a}^{\infty}\left[r \log r \psi(\varepsilon R(r)) p_{M}(r)-\frac{f(r)}{4 r \log r}\right] d r=+\infty
$$

for all $\varepsilon>0$, where $p_{M}(r)$ is given by (8) and $f(r)$ is defined in $\S 2$.

THEOREM 13. Under the assumptions of Theorem 10, the superlinear inequality $u L(u ; x) \leq 0$ is oscillatory in an exterior domain $\Omega$ of $R^{n}, n \geqslant 3$, if (10) holds and there exists a positive number a such that

$$
\int_{a}^{\infty}\left[r \psi(\varepsilon R(r)) p_{M}(r)-\frac{(n-2)^{2} f(r)}{r}\right] d r=+\infty
$$

for all $\varepsilon>0$. 
In the special case of the Emden-Fowler equation (14), $L_{0}(u ; x)=(\Delta u)(x)$, and one can check that $R(r)$ in Lemma 9 is given by $R(r)=(a / r)^{n-2}, n \geq 2$. In the superlinear case, $\gamma>1$ in (14) and we assume as usual that $\gamma$ is a quotient of odd positive integers in order to have $\phi(-t)=(-t)^{\gamma}=-\phi(t)$ for all $t>0$, as required in assumption $\left(\mathrm{B}_{1}\right)$. Then $\psi(t)=\phi(t) / t=t^{\gamma-1}$, and for $f(r)=1, n=2$, and $\psi\left(\varepsilon_{1} R(r)\right)=\varepsilon_{1}^{\gamma-1}=\varepsilon,(25)$ specializes to

$$
\int_{a}^{\infty}\left[\varepsilon r \log r p_{M}(r)-\frac{1}{4 r \log r}\right] d r=+\infty
$$

for all $\varepsilon>0$. Similarly for $f(r)=1, n \geq 3$, and $\psi\left(\varepsilon_{1} R(r)\right)=\varepsilon r^{(2-n)(\gamma-1)}$, where $\varepsilon=\left(\varepsilon_{1} a^{n-2}\right)^{\gamma-1},(26)$ specializes to

$$
\int_{a}^{\infty}\left[\varepsilon r^{\sigma} p_{M}(r)-\frac{(n-2)^{2}}{r}\right] d r=+\infty
$$

for all $\varepsilon>0$, where $\sigma=(n-1)-\gamma(n-2), n \geq 3$. It can be seen that (27) and (28) are quite sharp oscillation criteria by comparison with the necessary and sufficient conditions for oscillation in the next section.

5. Oscillation criteria by spherical means. Sharper criteria than (27) and (28) were obtained in [28], under the slightly stronger hypotheses (SL) below:

$$
B(x, t) \geq P_{1}(|x|) \phi(t) \text { for all } x \in \Omega, \quad t \geq 0,
$$

where $P_{1}$ is continuous and nonnegative in $[0, \infty)$, and $\phi$ is a continuously differentiable convex function in $[0, \infty)$ with $\phi(t)>0$ and $\phi^{\prime}(t) \geq 0$ in $(0, \infty)$. This requires slightly more than the union of $\left(\mathrm{B}_{2}\right),\left(\mathrm{SL}_{1}\right)$, and $\left(\mathrm{SL}_{2}\right)$, and actually could be weakened somewhat [28]. The method in [28] associates every solution $u(x)$ of (13) (or (14)) with its spherical mean $u_{M}(r)$ over the sphere $S_{r}$ of radius $r$, as defined by (8):

$$
u_{M}(r)=\frac{1}{s\left(S_{r}\right)} \int_{S_{r}} u(x) d s=\frac{1}{\omega\left(S_{1}\right)} \int_{S_{1}} u(x) d \omega .
$$

LEMMA 14. If $f: G_{a} \rightarrow R^{1}$ is a $C^{1}\left(G_{a}\right)$ function for some number $a>0$, then

$$
\int_{S_{r}}\left(D_{i} f\right)(x) d s=\frac{d}{d r} \int_{S_{r}} f(x) \frac{x_{i}}{r} d s
$$

for $i=1,2, \ldots, n, a<r<\infty$.

LEMMA 15. Under assumptions $\left(\mathrm{B}_{1}\right)$ and $(\mathrm{SL})$, the spherical mean $u_{M}(r)$ of a positive-valued solution $u \in C^{2}\left(G_{a}\right)$ of (13) satisfies the ordinary differential inequality

$$
\left[r^{n-1} u_{M}^{\prime}(r)\right]^{\prime}+r^{n-1} P_{1}(r) \phi\left[u_{M}(r)\right] \leq 0
$$

for $a<r<\infty$. 
The idea of the proof of Lemma 15 [28] is to use Lemma 14 twice to verify by direct calculation that $u_{M}(r)$ satisfies

$$
\left[r^{n-1} u_{M}^{\prime}(r)\right]^{\prime}=-\frac{1}{\omega\left(S_{1}\right)} \int_{S_{r}} B(x, u(x)) d s .
$$

Then (29) follows upon application of Jensen's inequality for convex functions $[32$, p. 160$]$ to $\phi[u(x)]$ on $S_{r}$ :

$$
\begin{aligned}
& \phi\left[u_{M}(r)\right]=\phi\left[\frac{1}{s\left(S_{r}\right)} \int_{S_{r}} u(x) d s\right] \leq \frac{1}{s\left(S_{r}\right)} \int_{S_{r}} \phi[u(x)] d s ; \\
& \int_{S_{\mathrm{r}}} B(x, u(x)) d s \geq P_{1}(r) \int_{S_{\mathrm{r}}} \phi[u(x)] d s \\
& \geq P_{1}(r) s\left(S_{r}\right) \phi\left[u_{M}(r)\right] \\
& =\omega\left(S_{1}\right) r^{n-1} P_{1}(r) \phi\left[u_{M}(r)\right]
\end{aligned}
$$

where (SL) has been used, and hence (30) implies (29).

THEOREM 16. Under assumptions $\left(\mathrm{B}_{1}\right)$ and (SL) the Schrödinger equation (13) is oscillatory in an exterior domain $\Omega$ in $R^{n}$ if the ordinary differential inequality (29) has no solution $u_{M}(r)$ which is positive throughout $\left[r_{0}, \infty\right)$ for any positive number $r_{0}$.

This is an easy consequence of Lemma 15, and reduces the oscillation problem for (13) to an analogous problem for the ordinary differential inequaltiy (29). This constitutes considerable progress since (29) is much easier to analyse directly than (13), and in fact special cases of (29) have been studied in detail for some time, see e.g. [13, 42, 44] for extensive bibliographies. Various sufficient conditions for (29) to have no eventually positive solution as $r \rightarrow \infty$ also are developed in [28], and applied via Theorem 16 to yield the following oscillation theorems for (13) and (14).

THEOREM 17. Under hypotheses $\left(\mathrm{B}_{1}\right)$ and (SL), the Schrödinger equation (13) is oscillatory in an exterior domain $\Omega$ in $R^{2}$ if there exists a positive number $c$ such that both

$$
\int^{\infty} \frac{d u}{\phi(u)}<\infty
$$

and

$$
\int_{c}^{\infty} r \log r P_{1}(r) d r=+\infty
$$

THEOREM 18. In the Emden-Fowler equation (14) suppose that $\gamma>1$, that $\gamma$ is a quotient of odd positive integers, and that $P_{1}(r)$ is continuous and nonnegative 
in $[0, \infty)$, where $P_{1}(r)=\min p(x)$ on the sphere $|x|=r$. Then a sufficient condition for (14) to be oscillatory in an exterior domain $\Omega$ in $R^{n}, n \geq 3$, is

$$
\int_{c}^{\infty} r^{\sigma} P_{1}(r) d r=+\infty
$$

for some $c>0$, where $\sigma=n-1-\gamma(n-2), \gamma>1$.

Modifications of Theorem 18 given in [28] apply to the Schrödinger equation (13).

Examination of the sufficient conditions (17) and (18) (of Theorem 8) for (14) to be nonoscillatory shows that (32) and (33) are very close to characterizations of oscillatory equations (14) in $R^{2}$ and $R^{n}, n \geq 3$, respectively. In fact, (32) and (33) are characterizations of oscillatory equations (14) under the additional hypothesis

$$
\liminf _{r \rightarrow \infty} P_{1}(r) / P(r)>0
$$

This condition merely restricts $p(x)$ from oscillating too severely on the sphere $S_{r}$ of radius $r$ for all sufficiently large $r$.

THEOREM 19. Suppose that $p(x)$ in (14) is nonnegative in an exterior domain $\Omega \subset R^{n}, p \in C^{\alpha}(\bar{M})$ for every bounded domain $M \subset \Omega, P \in C^{\alpha}[a, b]$ for all $b>a$, $a>0,0<\alpha<1$, and (34) is satisfied. Then (32) or (33) is necessary and sufficient for a superlinear equation (14) to be oscillatory in $\Omega$ according as $n=2$ or $n \geq 3$, respectively.

Since the hypotheses of Theorem 19 certainly imply those of Theorem 17 (specialized to the Emden-Fowler case (14)) and Theorem 18, the sufficiency of (32) and (33) follow from those theorems.

Conversely, if (14) is oscillatory in $\Omega$, then Theorem 8 shows that the integrals on the left sides of (17) and (18) diverge to $+\infty$. By (34) there exist positive numbers $r_{0}$ and $\varepsilon$ such that $P_{1}(r) \geq \varepsilon P(r)$ whenever $r \geq r_{0}$, and consequently

$$
\int_{\dot{r}_{0}}^{\infty} r \log r P_{1}(r) d r \geq \varepsilon \int_{r_{0}}^{\infty} r \log r P(r) d r=+\infty,
$$

proving the necessity of (32), and similarly the necessity of (33).

6. Sublinear equations. Oscillation and nonoscillation criteria for (13) and (14) will be described under assumptions $\left(B_{1}\right),\left(B_{2}\right)$, and the sublinear hypothesis below:

(SB) $\phi(t)$ in $\left(\mathrm{B}_{2}\right)$ satisfies $\phi^{\prime}(t)>0$ and $\Phi(t)<\infty$ for all $t>0$, where

$$
\Phi(t)=\int_{0}^{t} \frac{d s}{\phi(s)} .
$$


In particular the Emden-Fowler equation (14) satisfies (SB) in the case $0<\gamma<1$.

THEOREM 20. If $\left(\mathrm{B}_{1}\right),\left(\mathrm{B}_{2}\right)$, and $(\mathrm{SB})$ are satisfied, a sufficient condition for the sublinear inequality $u L(u ; x) \leq 0$ (given by (13)) to be oscillatory in an exterior domain $\Omega \subset R^{n}(n \geq 2)$ is

$$
\int_{c}^{\infty} r p_{M}(r) d r=+\infty
$$

for some $c>0$, where the spherical mean $p_{M}(r)$ of $p(x)$ over $S_{r}$ is defined by (8).

The proof given by Kitamura and Kusano [18] for the special case $L(u ; x)=$ $\Delta u+p(x) \phi(u)$ can be extended to (13). A different proof for $n=2$ was given in [29], based on the Riccati inequality (23). Neither $B(x, t)$ nor $p(x)$ is required to be everywhere positive for Theorem 20 to be true.

Sharp nonoscillation criteria for (14) in the sublinear case $0<\gamma<1$ are obtained on the basis of Lemma 7; these are stated in the theorem below.

THEOREM 21 [31]. Under the hypotheses on $p$ and $P$ in Lemma 7, a sufficient condition for the sublinear (i.e. $0<\gamma<1$ ) Emden-Fowler equation (14) to be nonoscillatory in an exterior domain $\Omega$ in $R^{n}$ is

$$
\begin{gathered}
\int_{c}^{\infty} r(\log r)^{\gamma} P(r) d r<\infty \quad \text { if } n=2, \\
\int_{c}^{\infty} r P(r) d r<\infty \quad \text { if } n \geq 3
\end{gathered}
$$

for some $c>0$.

It is interesting to compare (36) and (37) with the corresponding criteria (17) and (18) in the superlinear case $(\gamma>1)$, as stated in Theorem 8 . The proof is very similar to that of Theorem 8 , except that Atkinson's criterion (20) is replaced by Belohorec's criterion [8, 44]

$$
\int_{s_{0}}^{\infty} s^{\gamma} e^{2 s} P\left(e^{s}\right) d s<\infty, \quad s=\log r
$$

(when $n=2$ ), which is equivalent to (36). The argument (for $n=2$ ) in Theorem 8 is then essentially unchanged.

Combination of Theorems 20 and 21 yields a necessary and sufficient condition for the sublinear equation (14) to be oscillatory in $\Omega \subset R^{n}, n \geq 3$ provided hypothesis (34) is added, thereby extending Belohorec's onedimensional characterization of sublinear oscillation to dimensions $n \geq 3$. However, the case $n=2$ is still unresolved.

THEOREM 22. If $p$ and $P$ satisfy the hypotheses of Theorem 19 and if (34) 
holds, then a necessary and sufficient condition for the sublinear (i.e. $0<\gamma<1$ ) Emden-Fowler equation (14) to be oscillatory in an exterior domain $\Omega \subset R^{n}$, $n \geq 3$ is

$$
\int_{c}^{\infty} r P(r) d r=+\infty
$$

for some positive number $c$.

The oscillation criteria (32), (33), (39), etc. for (14) have the form (where $\gamma$ is a quotient of odd positive integers)

$$
\int_{c}^{\infty} m(r) P_{1}(r) d r=+\infty
$$

for some radial function $m$ and some number $c>0$, where $P_{1}(r)=\min p(x)$ on the sphere $|x|=r$. Under the hypotheses associated with Theorems 19,22 , etc., some of these criteria are characterizations of oscillatory equations (14). A summary of the known results is tabulated below according to dimension $n$ and semilinear exponent $\gamma$. All the nonlinear entries correspond to necessary and sufficient conditions for oscillation, except the entry for $n=2,0<\gamma<1$.

\begin{tabular}{|c|c|c|c|}
\hline Dimension & Sublinear $(0<\gamma<1)$ & Linear $(\gamma=1)$ & Superlinear $(\gamma>1)$ \\
\hline 1 & $\begin{array}{l}r^{\nu} \\
\text { NASC, } 1961 \\
\text { Belohorec [8] }\end{array}$ & $\begin{array}{l}r^{b}, b<1 \\
1955 \\
\text { Moore [25] }\end{array}$ & $\begin{array}{l}r \\
\text { NASC, } 1955 \\
\text { Atkinson [7] }\end{array}$ \\
\hline 2 & $\begin{array}{l}r \\
(35), 1979 \\
\text { Kitamura and Kusano [18] }\end{array}$ & $\begin{array}{l}r(\log r)^{b}, b<1 \\
(11), 1975 \\
\text { Noussair and I [28] }\end{array}$ & $\begin{array}{l}r \log r \\
\text { NASC (32), } 1979 \\
\text { Noussair and I [30] }\end{array}$ \\
\hline$n \geq 3$ & $\begin{array}{l}r \\
\text { NASC, (39), } 1979 \\
\text { Noussair and I [31] }\end{array}$ & $\begin{array}{l}r^{b}, b<1 \\
(6), 1968 \\
\text { Headley and I [16] }\end{array}$ & $\begin{array}{l}r^{\sigma}, \sigma=n-1-\gamma(n-2) \\
\text { NASC (33), 1979 } \\
\text { Noussair and I [30] }\end{array}$ \\
\hline
\end{tabular}

TABLE OF MULTIPLIERS $m(r)$ FOR OSCILLATION OF (14)

7. Perturbed linear equations. The elliptic equation (1) is called a perturbed linear equation when $B(x, u)$ in (1) has the form

$$
B(x, u)=q_{1}(x) u+\sum_{j=2}^{J} q_{j}(x) \psi_{j}(u), \quad x \in \Omega
$$

under the assumptions $\left(\mathrm{PL}_{1}\right)$ and $\left(\mathrm{PL}_{2}\right)$ below:

$\left(\mathrm{PL}_{1}\right)$ Each $q_{\mathrm{j}}$ is a continuous real-valued function in $\Omega, \mathrm{j}=1,2, \ldots, J$;

$\left(\mathrm{PL}_{2}\right)$ Each $\psi_{j}$ is an odd $C^{1}$ function in $(-\infty, \infty)$ with $\psi_{j}(t)>0$ for all $t>0$. The functions $q_{j}$ are not required to be everywhere positive, precluding the 
deduction of oscillation criteria via comparison theorems for $(1)[37,41]$. We define functions $p$ and $\phi$ by

$$
\begin{aligned}
p(x) & =\min \left[q_{1}(x), \ldots, q_{J}(x)\right], \quad x \in \Omega \\
\phi(u) & =u+\sum_{j=2}^{J} \psi_{j}(u), \quad-\infty<u<\infty .
\end{aligned}
$$

Then $B(x, u) \geq p(x) \phi(u)$ for all $x \in \Omega$ and for all $u \geq 0$, and hence $\left(\mathrm{B}_{1}\right)$ and $\left(\mathrm{B}_{2}\right)$ in $\S 1$ are implied by $\left(\mathrm{PL}_{1}\right)$ and $\left(\mathrm{PL}_{2}\right)$. The following oscillation criteria for a perturbed linear equation (1) or inequality $u L(u ; x) \leq 0$ are proved in [29] by methods similar to those described in $\$ 4$ above.

THEOREM 23. The perturbed linear inequality $u L(u ; x) \leq 0$ (given by (1) and (40)) is oscillatory in an exterior domain $\Omega$ of $R^{2}\left(R^{n}, n \geq 3\right.$, respectively) under hypotheses $(\mathrm{A}),\left(\mathrm{PL}_{1}\right)$ and $\left(\mathrm{PL}_{2}\right)$ if $(9)$ and $(11)((10$ and $(12)$, respectively) are satisfied, where $p(x)$ is given by (41).

For example, if $\left(A_{i j}\right)$ in (1) is a constant matrix with largest eigenvalue $\lambda$, oscillation criteria for (1), (40) are

$$
\begin{array}{rr}
\int_{a}^{\infty}\left[r \log r p_{M}(r)-\frac{\lambda}{4 r \log r}\right] d r=+\infty, & n=2, \\
\int_{a}^{\infty}\left[r p_{M}(r)-\frac{(n-2)^{2} \lambda}{r}\right] d r=+\infty, & n \geq 3,
\end{array}
$$

for some $a>0$, where $p_{M}(r)$ is given by (8), (41).

8. Oscillation in general domains. The foregoing theory applies essentially only to unbounded domains $\Omega$ of the exterior type. In the case of the linear equation (2), sufficient conditions for (nodal) oscillation were obtained in [40] without special hypotheses on the geometry of $\Omega$. These results are written in terms of the functional

$$
F[u ; M]=\int_{M}\left[\sum_{i, j=1}^{n} A_{i j}(x) D_{i} u D_{j} u-p(x) u^{2}\right] d x
$$

on a nonempty regular bounded domain $M \subset \Omega$, where the admissible functions $u$ in (42) are supposed to be real-valued piecewise $C^{1}$ functions on $\bar{M}$.

THEOREM 24. Equation (2) is nodally oscillatory in an unbounded domain $\Omega \subset R^{n}$ if $\Omega$ contains a sequence of nonempty regular bounded domains $M_{k}$, $k=1,2, \ldots$, with piecewise $C^{1}$ boundaries, having the following properties:

(i) For arbitrary $r>0$ there exists an integer $k_{0}(r)$ such that $M_{k} \subset \Omega_{r}$ for all $k \geq k_{0}(r) ;$ and

(ii) There exists a nontrivial piecewise $C^{1}$ function $u_{k}$ on each $M_{k}$ such that 
$u_{k}(x)=0$ for all $x \in \partial M_{k}$ and $F\left[u_{k} ; M_{k}\right] \leq 0$ whenever $k \geq K$ for some positive integer $K$.

This is proved in [40] by Courant's minimum principle for eigenvalues [11, $p$. 399]. We can prove, furthermore, under the same hypotheses, that the linear inequality $v L(v ; x) \leq 0$ is oscillatory in $\Omega$ by appealing to Picone's identity [39, 41]. In fact, if $v$ is a positive or negative solution of the inequality throughout $\bar{M}_{k}$ for $k \geq \max \left(k_{0}(r), K\right)$, integration of Picone's identity over $M_{k}$ yields the contradiction

$$
\int_{M_{k}} \frac{u_{k}^{2}(x)}{v(x)} L(v ; x) d x+F\left[u_{k} ; M_{k}\right]>0
$$

unless $v$ is a constant multiple of $u_{k}$ in $\bar{M}_{k}$. Therefore every solution $v$ of $v L(v ; x) \leq 0$ has a zero in $\Omega_{r}$ for all $r>0$. Nodal oscillation of (2) follows from Allegretto's equivalence theorem [2] (see §1) if $p \in C^{\alpha}$ and each $A_{i j} \in C^{\alpha+1}$, where

$$
\alpha=3\left[\frac{1}{2}\left[\frac{n+6}{2}\right]\right] .
$$

THEOREM 25. Equation (2) is nodally oscillatory in an unbounded domain $\Omega \subset R^{n}$ if $\Omega$ contains a sequence of disks $M_{k}\left(x_{k} ; a_{k}\right)=\left\{x:\left|x-x_{k}\right|<a_{k}\right\}$ with the following properties:

(i) $\lim _{k \rightarrow \infty}\left(\left|x_{k}\right|-a_{k}\right)=+\infty$;

(ii) $\left(A_{i j}(x)\right)$ is bounded in $\bigcup_{k} M_{k}\left(x_{k} ; a_{k}\right)$; and

(iii) $\lim _{k \rightarrow \infty} a_{k}^{2-n} \int_{M_{k}\left[x_{k} ;(1 / 2) a_{k}\right]} p(x) d x=+\infty$.

This is deduced from Theorem 24 if $u_{k}$ is selected to be the piecewise linear function of $r=\left|x-x_{k}\right|$ which is 1 in $0 \leq r \leq a_{k} / 2$ and 0 for $r \geq a_{k}$. Various specializations of Theorem 25 lead to nodal oscillation criteria for (2) in domains $\Omega$ which are quasiconical, quasicylindrical, or even of the "spiral" type containing no infinite ray. These results also have been extended by Noussair [27] and Allegretto [5] to certain elliptic operators of arbitrary even order.

Allegretto [4] has obtained results of similar type for semilinear equations (1) under the following geometric assumptions: (i) As in Theorem 24, there exists an integer $k_{0}$ such that $M_{k} \subset \Omega_{r}$ for all $k \geq k_{0}$; (ii) As in Theorem $25, M_{k}$ is specialized to an $n$-disk $M_{k}\left(x_{k} ; a_{k}\right)$ of radius $a_{k}$ and centre $x_{k}$; and (iii) For each integer $k=1,2, \ldots, x_{k+1} \in M_{k}, M_{k+1} \not M_{k}$, and $x_{k} \neq x_{j}$ if $k \neq j$. Allegretto defines a function $g_{k}$ in $M_{k}\left(x_{k} ; \frac{1}{2} a_{k}\right), k=1,2, \ldots$, depending on the geometry and the coefficient functions in (1) in a complicated way; then, if $\left(A_{i j}(x)\right)$ is bounded (as a form) in $\Omega$, a sufficient condition for (1) to be oscillatory in $\Omega$ is that

$$
\lim _{k \rightarrow \infty}\left\{a_{k}^{2-n} \int_{M_{k}\left[x_{k} ;(1 / 2) a_{k}\right]} F\left(x ; \varepsilon g_{k}(x)\right) d x\right\}=+\infty
$$


for every positive number $\varepsilon$, where $F(x, t)=t^{-1} B(x, t)$. Illustrations of this criterion are given in [4] when (1) reduces to the Emden-Fowler equation (14) in unbounded domains of various specific types.

\section{REFERENCES}

1. S. Agmon, A. Douglis, and L. Niremberg, Estimates near the boundary for solutions of elliptic partial differential equations satisfying general boundary conditions, Comm. Pure Appl. Math. XII (1959), 623-727.

2. W. Allegretto, On the equivalence of two types of nonoscillation for elliptic operators, Pacific J. Math. 55 (1974), 319-328.

3. - Oscillation criteria for quasilinear equations, Canad. J. Math. 26 (1974), 931-947.

4. - Oscillation criteria for semilinear equations in general domains, Canad. Math. Bull. 19 (1976), 137-144.

5. - A Kneser theorem for higher order elliptic equations, Canad. Math. Bull. 20 (1977), 1-8.

6. H. Amann, On the existence of positive solutions of nonlinear elliptic boundary value problems, Indiana Univ. Math. J. 21 (1971), 125-146.

7. F. V. Atkinson, On second order nonlinear oscillations, Pacific J. Math. 5 (1955), 643-647.

8. S. Belohorec, Oscillatory solutions of certain nonlinear differential equations of second order, Mat.-Fyz. Casopis Sloven. Akad. Vied. 11 (1961), 250-255.

9. - On some properties of the equation $y^{\prime \prime}(x)+f(x) y^{\alpha}(x)=0,0<\alpha<1$, Mat.-Fyz. Casopis Sloven. Akad. Vied. 17 (1967), 10-19.

10. W. J. Coles, A simple proof of a well-known oscillation theorem, Proc. Amer. Math. Soc. 19 (1968), 507.

11. R. Courant and D. Hilbert, Methods of Mathematical Physics I, Wiley (Interscience), New York, 1953.

12. _ - Methods of Mathematical Physics II, Wiley (Interscience), New York-London, 1962.

13. L. Erbe, Oscillation criteria for second order nonlinear differential equations, Ann. Mat. Pura Appl. 94 (1972), 257-268.

14. I. M. Glazman, Direct Methods of Qualitative Spectral Analysis of Singular Differential Operators, Israel Program for Scientific Translations, Daniel Davey and Co., New York, 1965.

15. V. B. Headley, Some oscillation properties of selfadjoint elliptic equations, Proc. Amer. Math. Soc. 25 (1970), 824-829.

16. V. B. Headley and C. A. Swanson, Oscillation criteria for elliptic equations, Pacific J. Math. 27 (1968), 501-506.

17. J. G. Heywood, E. S. Noussair, and C. A. Swanson, On the zeros of solutions of elliptic inequalities in bounded domains, J. Differential Equations 28 (1978), 345-353.

18. Y. Kitamura and T. Kusano, Oscillation of second order sublinear elliptic equations, Utilitas Math., to appear.

19. K. Kreith, Oscillation theorems for elliptic equations, Proc. Amer. Math. Soc. 15 (1964), 341-344.

20. _ Oscillation Theory, Lecture Notes in Mathematics, Vol. 324, Springer Verlag, Berlin, 1973.

21. K. Kreith and C. C. Travis, Oscillation criteria for self-adjoint elliptic differential equations, Pacific J. Math. 41 (1972), 743-753.

22. L. M. Kuks, Sturm's theorem and oscillation of solutions of strongly elliptic systems, Soviet Math. Dokl. 3 (1962), 24-27.

23. O. A. Ladyzhenskaya and N. N. Ural'tseva, Linear and Quasilinear Elliptic Equations, Academic Press, New York, 1968.

24. W. Leighton, On self-adjoint differential equations of second order, J. London Math. Soc. 27 (1952), 37-47.

25. R. A. Moore, The behavior of solutions of a linear differential equation of second order, Pacific J. Math. 5 (1955), 125-145. 
26. Z. Nehari, On a class of nonlinear second-order differential equations, Trans. Amer. Math. Soc. 95 (1960), 101-123.

27. E. S. Noussair, Oscillation of elliptic equations in general domains, Canad. J. Math. 27 (1975), 1239-1245.

28. E. S. Noussair and C. A. Swanson, Oscillation theory for semilinear Schrödinger equations and inequalities, Proc. Roy. Soc. Edinburgh, Sect. A, 75 (1975/76), 67-81.

29. — Oscillation of semilinear elliptic inequalities by Riccati transformations, Canad. J. Math., to appear.

30. —_ Positive solutions of semilinear Schrödinger equations in exterior domains, Indiana Univ. Math. J. (submitted).

31. - Positive solutions of quasilinear equations in exterior domains, under preparation.

32. G. O. Okikiolu, Aspects of the Theory of Bounded Integral Operators in $L^{p}$-spaces, Academic Press, New York, 1971.

33. J. Piepenbrink, Nonoscillatory elliptic equations, J. Differential Equations 15 (1974), 541550.

34. —- A conjecture of Glazman, J. Differential Equations 24 (1977), 173-177.

35. M. H. Protter and H. F. Weinberger, Maximum Principles in Differential Equations, Prentice-Hall, Englewood Cliffs, N.J., 1967.

36. W. T. Reid, Riccati Differential Equations, Mathematics in Science and Engineering, Vol. 86, Academic Press, New York-London, 1972.

37. C. A. Swanson, Comparison and Oscillation Theory of Linear Differential Equations, Mathematics in Science and Engineering, Vol. 48, Academic Press, New York, 1968.

38. _ - Nonoscillation criteria for elliptic equations, Canad. Math. Bull. 12 (1969), 275-280.

39. - Remarks on Picone's identity and related identities, Atti Accad. Naz. Lincei Mem. Cl.

Sci. Fis. Mat. Natur., Sez. VIII, 11 (1972), 1-15.

40. - Strong oscillation of elliptic equations in general domains, Canad. Math. Bull. 16 (1973), 105-110.

41. —, Picone's identity, Rend. Mat. (2) 8 (1975), 373-397.

42. D. Willett, On the oscillatory behavior of the solutions of second order linear differential equations, Ann. Polon. Math. 21 (1969), 175-194.

43. A. Wintner, A criterion of oscillatory stability, Quart. Appl. Math. 7 (1949), 115-117.

44. James S. W. Wong, On the generalized Emden-Fowler equation, SIAM Rev. 17 (1975), 339-360.

\section{DEPARTMENT OF MATHEMATICS \\ UNIVERSITY OF BRTTISH COLUMBIA \\ VANCOUVER, B.C. V6T 1W5}

